\title{
OBSERVATORIO GALLEGO DE E-LEARNING: METODOLOGÍA, TÉCNICAS E INSTRUMENTOS
}

(GALICIAN E-LEARNING OBSERVATORY: METHODOLOGY, SKILLS AND INSTRUMENTS)

\author{
Manuel Gromaz \\ Javier García Tobío \\ María José Rodríguez \\ Miguel Beremejo \\ Centro de Supercomputación de Galicia (España) \\ Carmen Fernández Morante \\ Beatriz Cebreiro López \\ Universidad de Santiago de Compostela (España) \\ María Isabel Doval Ruíz \\ Universidad de Vigo (España) \\ Marta Fernández Prieto \\ Universidad de A Coruña (España)
}

\section{RESUMEN}

Para llevar a cabo el proyecto de investigación denominado Observatorio gallego de e-learning, se diseño una metodología de estudio acorde a las características de la población a analizar sin perder de vista el objetivo final: analizar el estado del e-learning en la empresa y en la universidad gallega.

En este artículo damos a conocer la metodología de investigación, las técnicas y los instrumentos utilizados para realizar el estudio tanto en la universidad (en la que se encuestó a Personal docente e investigador y se entrevistó a órganos directivos, directores de grupos de investigación y administradores de los campus virtuales) como en la empresa (en la que se encuestó telefónicamente a empresas demandantes de e-learning y se realizó un cuestionario a empresas ofertantes de e-learning).

Palabras clave: e-learning, observatorio, TIC, profesores, empresa. 


\begin{abstract}
To carry out the investigation project called Galician e-learning observatory, a study's methodology was designed accord to the characteristics of the population for analyze without losing the final objective: analyze the state of the art of the e-learning in the Galician company and in the Galician university.

In this article we announce the methodology of the investigation, the skills and the instruments used for make the study so much in the university as in the company.
\end{abstract}

Key words: e-learning, observatory, ICT, teachers, company.

El Observatorio gallego de e-learning es un proyecto de investigación que tiene como principales objetivos la monitorización científica y la evaluación del uso de las Tecnologías de la Información y la Comunicación en el ámbito de la educación y la formación en Galicia, mediante la catalogación y análisis de las prácticas existentes, servicios disponibles, agentes públicos y privados que desarrollan una labor en el campo de la investigación e innovación en el área de e-learning en Galicia. Así mismo, tratará de convertirse en el referente para el conocimiento de prácticas innovadoras que se desarrollen en el entorno gallego en cuanto a investigación metodológica, tecnologías aplicadas a la educación, agentes públicos y privados del sector, y como punto de encuentro dinamizador para los expertos en e-learning de nuestra comunidad.

\title{
Población y muestra objeto de estudio
}

El estudio definió una población y muestra a analizar en el caso de la universidad, y de la empresa. En este artículo sólo vamos abordar la parte de la investigación realizada en las tres universidades gallegas, y dejamos para otro lo concerniente a la empresa.

Población y muestra objeto de estudio en la universidad. Procedimiento de selección

Para analizar el estado del e-learning en las universidades gallegas, en este estudio se definieron los siguientes segmentos poblacionales:

- Personal docente e investigador de las tres universidades gallegas.

- Órganos directivos de las universidades gallegas.

- Administradores y asesores pedagógicos de los Campus Virtuales de las universidades gallegas. 
- Grupos de investigación que llevan a cabo o están relacionados con actividades de e-learning en las universidades gallegas.

- Personal docente e investigador de las tres universidades gallegas: Se consideró como segmento poblacional a todo el Personal Docente e Investigador (PDI) de las tres Universidades de Galicia, para tratar de conocer no sólo si los docentes utilizan las TIC en la enseñanza, sino también qué tecnologías utilizan, cómo y para qué las utilizan.

Para definir la muestra objeto de estudio en el caso del profesorado, utilizamos un muestreo probabilístico aleatorio simple.

En la siguiente tabla se puede ver la población total de profesorado universitario en Galicia para el curso académico 2004-2005 en su totalidad, así como para cada universidad en particular:

\begin{tabular}{|c|c|c|c|c|}
\hline & $\mathrm{N}^{1}$ & $\% \mathrm{~N}^{2}$ & $\mathrm{n}^{3}$ & $\% \mathrm{n}^{4}$ \\
\hline USC & 2182 & 42,4 & 382 & $44,8 \%$ \\
\hline UDC & 1326 & 25,7 & 250 & $29,3 \%$ \\
\hline UVIGO & 1640 & 31,8 & 221 & $25,9 \%$ \\
\hline Total & 5148 & 99,9 & 897 & $100 \%$ \\
\hline
\end{tabular}

Tabla 1: Tabla poblacional y muestral de personal docente e investigador correspondiente a las tres universidades gallegas para el curso académico 2004-2005.

Como se aprecia en la tabla anterior, la muestra objeto de estudio estuvo formada por profesorado de las tres universidades gallegas, esto es, A Coruña, Santiago de Compostela y Vigo de una población total de 5.148 profesores.

Atendiendo a un muestreo aleatorio simple, calculamos el margen de error en las respuestas, el cual, atendiendo a un nivel de confianza del 95\% de la totalidad de la muestra, es tan sólo de +-3, mientras que si en lugar de ser un estudio aleatorio simple, fuera estratificado por ejemplo atendiendo al tipo de universidad, tendríamos un margen de error variable atendiendo a cada institución de enseñanza superior. 


\begin{tabular}{|ccccc|}
\hline Universidad & Población & $\begin{array}{c}\text { Tamaño de la } \\
\text { muestra }\end{array}$ & $\begin{array}{c}\text { Nivel de } \\
\text { confianza }\end{array}$ & $\begin{array}{c}\text { Error } \\
\text { muestral }\end{array}$ \\
UDC & 1326 & 250 & $95 \%$ & $+-5,6$ \\
USC & 2182 & 382 & $95 \%$ & $+-4,6 \%$ \\
UVIGO & 1640 & 221 & $95 \%$ & $+-6,1 \%$ \\
Total & 5148 & 897 & $95 \%$ & $+-3 \%$ \\
\hline
\end{tabular}

Tabla 2: Tabla de cálculo de error muestral por universidad para un nivel de confianza del 95\%.

- Órganos directivos de las universidades gallegas: otro segmento objeto de análisis y definido en este estudio fueron los órganos directivos. Aunque también forman parte de los PDI de la universidad, se consideró relevante estudiar este colectivo de forma específica debido a que son los responsables de la toma de decisiones estratégicas que afectan al funcionamiento de la institución. Para ello se seleccionaron aquellos órganos directivos implicados directamente en políticas de e-learning en cada universidad para investigar mediante técnicas de investigación cualitativa.

En la siguiente tabla se puede apreciar el número de órganos directivos por universidad correspondientes al año 2005 y la muestra seleccionada para este estudio:

\begin{tabular}{|c|c|c|c|c|}
\hline Órganos directivos & UDC & USC & UVIGO & Total \\
\hline $\mathrm{N}$ & 10 & 9 & 9 & 28 \\
$\mathrm{n}$ & 1 & 1 & 2 & 4 \\
\hline
\end{tabular}

Tabla 3: Tabla poblacional y muestral de los Órganos directivos de las universidades gallegas.

- Administradores y asesores pedagógicos de los Campus Virtuales de las universidades gallegas: de igual modo, se consideró esencial investigar a los administradores y asesores pedagógicos de los Campus Virtuales (pues ellos son responsables delfuncionamientodelossistemas degestión delaprendizaje que utilizan alumnado y profesorado, así como los que recomiendan metodologías y dan pautas de utilización de los diferentes recursos) que nos facilitaron información no sólo del grado de utilización de los Sistemas de Gestión del Aprendizaje en cada institución universitaria, sino del tipo de profesorado y alumnado que hacen uso de estas herramientas. 
Para investigar este pequeño colectivo, dadas sus reducidas dimensiones, también se utilizó una metodología cualitativa.

\begin{tabular}{|c|c|c|c|c|}
\hline $\begin{array}{c}\text { Administradores y asesores } \\
\text { pedagógicos de los Campus } \\
\text { Virtuales }\end{array}$ & UDC & USC & UVIGO & Total \\
\hline $\mathrm{N}$ & 1 & 1 & 2 & 4 \\
$\mathrm{n}$ & 1 & 1 & 2 & 4 \\
\hline
\end{tabular}

Tabla 4: Tabla poblacional y muestral de los administradores de los Campus Virtuales.

Como se puede apreciar en la tabla anterior, se entrevistó al administrador del Campus Virtual de la Universidad de A Coruña, al administrador del Campus Virtual de la Universidad de Santiago de Compostela y a dos administradores del Campus Virtual de la Universidad de Vigo. El hecho de que en la Universidad de Vigo se realizaran dos entrevistas fue debido a que esta institución dispone de dos Campus Virtuales, uno orientado a cursos de postgrado y otro orientado a cursos de grado. En el caso de la Universidad A Coruña, también nos encontramos con la misma situación, pero los cursos de postgrado se imparten a través de un sistema de gestión del aprendizaje comercial gestionado por una empresa externa a la Universidad. En el momento del análisis, la Universidad de Santiago impartía toda la formación de grado y de postgrado a través del mismo sistema de gestión del aprendizaje.

- Grupos de investigación en Educación y Tecnologías de la Información y de la Comunicación en las universidades gallegas: otro segmento definido en la investigación, fueron los grupos de investigación en e-learning, ya que además de identificar estos grupos en las universidades gallegas, nos permitió conocer las líneas de investigación en e-learning, los tipos de proyectos que desarrollan, así como el tipo de profesionales que los integran. La metodología bajo la que se investigaron estos grupos fue la cualitativa.

\begin{tabular}{|c|c|c|c|c|}
\hline $\begin{array}{c}\text { Grupos de Investigación en } \\
\text { e-learning }\end{array}$ & UDC & USC & UVIGO & Total \\
\hline $\mathrm{N}$ & 5 & 6 & 8 & 19 \\
\hline $\mathrm{n}$ & 3 & 4 & 6 & 13 \\
\hline \% de Grupos investigados & $60 \%$ & $66,66 \%$ & $75 \%$ & $68,42 \%$ \\
\hline
\end{tabular}

Tabla 5: Tabla poblacional y muestral de grupos de investigación en e-learning o relacionados con e-learning. 
Para esta investigación se identificaron en las Oficinas de Transferencia de Resultados e Investigación (OTRI) de las tres universidades aquellos grupos que tenían como principal línea de investigación la utilización de las Tecnologías de la Información y de la Comunicación en la Educación. En este estudio, además, también se contemplaron aquellos grupos que contaban con alguna línea de investigación o disponían de palabras clave entre sus descriptores relacionadas con esta temática. De este modo, como se puede apreciar en la tabla anterior, nos pusimos en contacto con un total de 19 grupos de investigación, de ellos 13 confirmaron estar desarrollando o haber desarrollado en los últimos tres años una línea de investigación afín al e-learning. Sin embargo, como se podrá apreciar más adelante, de estos trece identificados, son muy pocos los grupos cuya principal actividad investigadora es el e-learning.

\section{Metodología de investigación utilizada}

El estudio llevado a cabo se trata de una investigación básica dirigida a obtener conocimiento básico mediante métodos descriptivos.

Partimos de la necesidad de aprovecharnos en este estudio, dado que se van a investigar numerosos colectivos (por lo tanto se requiere la utilización de numerosas muestras) pertenecientes a diferentes realidades, de las características principales de dos de los tres principales paradigmas en investigación educativa, el positivista basado en metodologías cuantitativas y el interpretativo que utiliza básicamente metodologías cualitativas. Entre los instrumentos de corte cuantitativo que se utilizaron están los cuestionarios, y entre las herramientas cualitativas la entrevista y el análisis documental.

Dicho todo esto, partimos por lo tanto de la "complementariedad entre paradigmas que aunque de base ontológica y epistemológica distinta, se apoyan y complementan en el proceso de investigación” (Bisquerra, 2004, p 77).

Siguiendo con el encuadre teórico de la investigación, se trata de un estudio ex post-facto con carácter descriptivo, concretamente dentro de los estudios de encuesta, puesto que utiliza como herramientas de recogida de información fundamentalmente el cuestionario y la entrevista. En la metodología cualitativa lo situamos en un estudio orientado a la comprensión, donde el método cualitativo utilizado es el etnográfico, más concretamente el estudio intrínseco de casos en el que utilizamos instrumentos de recogida de datos directos como las entrevistas e indirectos como el análisis documental de material público. 
Metodología e Instrumentos de recogida de información en el ámbito universitario

Para la recogida de datos así como para triangular la información y tomando como referencia las características de los segmentos poblacionales definidos para llevar a cabo la investigación a realizar, se utilizó una metodología mixta, en la que se utilizaron instrumentos propios de la metodología cuantitativa y herramientas propias de la metodología cualitativa. En la parte metodológica cuantitativa se utilizaron cuestionarios, mientras que en la cualitativa se llevaron a cabo entrevistas y análisis de fuentes públicas documentales. Se trata éste de un estudio, al igual que el de Cabero (2002), que permite la triangulación tanto de sujetos como de métodos, puesto que la triangulación de sujetos hace referencia a la obtención de información procedente de diferentes vías, en este caso de profesorado, órganos directivos, administradores de Campus Virtuales y grupos de investigación, y la triangulación metodológica, ya que se utiliza diferentes metodologías (cuantitativa y cualitativa) para la recogida de información (cuestionarios, entrevistas, fuentes públicas documentales).

Este planteamiento metodológico ayudó a profundizar en la investigación con la obtención de información procedente de numerosas fuentes. De esta forma en el análisis de datos se pudo triangular la información obtenida de diferentes fuentes y a través de diversos métodos tal y como se podrá ver en el análisis de datos.

Para la elaboración de las diferentes herramientas de investigación (entrevistas y cuestionarios), consultamos, entre otra, la documentación reseñada en la bibliografía.

La metodología utilizada y los instrumentos que elaboramos y que describimos a continuación se construyeron acorde a los objetivos establecidos y a las dimensiones y características de la población a investigar, esto es:

- Personal Docente e Investigador,

- Órganos Directivos,

- Administradores y Asesores pedagógicos de los Campus Virtuales y

- Grupos de investigación en e-learning. 
Técnicas e instrumentos utilizados para investigar el Personal docente e investigador

Para analizar la utilización de las TIC en la educación por parte del personal docente e investigador se usó una metodología cuantitativa que posibilitó la realización del estudio en una población que abarca a todos los docentes de las tres universidades gallegas y que estuvo compuesta por 5.148 individuos ${ }^{5}$. Para ello se decidió elaborar un cuestionario online debido a la magnitud de la población a investigar. Esta herramienta nos permitió hacer llegar el cuestionario de forma exclusiva a todos los docentes e investigadores de las tres universidades de Galicia.

El proceso de elaboración y diseño del cuestionario pasó por diferentes fases: revisión de la literatura científica sobre investigaciones realizadas acerca de la utilización de las TIC en la universidad, juicio de expertos y estudio piloto.

De esta forma el diseño de este cuestionario tomó como referencia una investigación realizada a nivel universitario coordinada por Julio Cabero Almenara (2002) y en la que también participó una profesora integrante de la mesa de expertos del proyecto. El cuestionario en un primer momento se diseñó como documento de texto entre los meses de diciembre de 2004 a enero de 2005. Una vez diseñado, y consensuado entre el panel, se remitió a tres expertos de reconocido prestigio a nivel nacional en Tecnología Educativa, a saber: Julio Cabero Almenara (Universidad de Sevilla), Pere Marqués Graells (Universidad Autónoma de Barcelona) y Miguel Zapata Ros (Universidad de Murcia). Aún con todas estas pruebas de validez superadas, se decidió enviar el cuestionario esta vez ya en formato web a un grupo piloto de profesores universitarios de fuera de Galicia para poder recoger sus impresiones y reproducir el proceso de cumplimentación del cuestionario en condiciones similares a como se haría en realidad.

Durante el mes de febrero de 2005 se diseñó el cuestionario online programado en lenguaje PHP alojado en un servidor Web Apache. Este cuestionario guardó la información introducida por los encuestados en un servidor de base de datos MySql. Todo ello instalado en un servidor Linux. Es decir, para el desarrollo del proyecto se utilizó tecnología LAMP (Linux, Apache, MySql y Php), es decir, aplicaciones de software libre adaptadas a los requerimientos del estudio. La decisión de utilizar esta tecnología ha sido su bajo costo, su flexibilidad de adaptación, su compatibilidad y su potencial tecnológico.

El cuestionario en formato online, dada su extensión y complejidad, se decidió diseñar en dos partes que se convirtieron en su versión digital en dos páginas Web; 
la primera parte del cuestionario recogió información general sobre el PDI de las universidades, mientras que la segunda parte del cuestionario respondía a cuestiones más específicas abordando cuestiones concretas sobre TIC y educación.

El cuestionario elaborado para el estudio constaba de 51 ítems que configuraron un total de 300 variables distribuidas en escalas nominales, ordinales y de razón, preguntas de respuesta abierta, etc.

En la primera parte del cuestionario, es decir, las preguntas comprendidas de la 1 a la 18, se incluyó un bloque de cuestiones de obligada respuesta, concretamente el intervalo de preguntas comprendidas entre la 12 y la 18, ya que, en función de que los docentes respondieran de forma afirmativa o negativa cada una de estas cuestiones, la segunda parte del cuestionario variaba en función de la respuesta otorgada. Esta segunda parte del cuestionario constaba de seis itinerarios, atendiendo a las siguientes preguntas (12-18) que conformaban las seis grandes dimensiones en torno a las cuales se había diseñado el cuestionario:

- Módulo I: Aspectos generales y personales del profesorado. Abarca las preguntas de la 1 a la 18.

- Módulo II: Utilización de las TIC en la docencia. Comprende las preguntas de la 19 a la 30 y la 43 .

- Módulo III: Utilización de las TIC en las tutorías. Engloba las mismas preguntas que el Módulo II.

- Módulo IV: Utilización de las TIC para formarse: sólo contempla la pregunta 14.

- Módulo V: Formación del profesorado para utilizar las TIC. Comprende las preguntas 31-34.

- Módulo VI: Utilización de contenidos en formato digital en la docencia. Integrado por las preguntas 35-37.

- Módulo VII: Realizar proyectos o formar parte de grupos de investigación en e-learning. Engloba las preguntas de la 38 a la 42.

De esta forma, el cuestionario online quedó conformado en seis itinerarios invisibles (puesto que el módulo I era común para todos) para el docente que los cumplimentaba. Mientras que el itinerario más largo fue para el docente que respondía afirmativamente a las preguntas 12-18, el camino más corto, por contraposición, era para el profesor que respondió negativamente a todas estas cuestiones. Estos itinerarios se corresponden con las grandes dimensiones del cuestionario: aspectos generales, utilización de las TIC en la docencia, formación en TIC, formación con TIC, diseño de contenidos digitales didácticos e investigación en TIC y Educación. 
El cuestionario online, después de someterlo a numerosas pruebas de funcionamiento tanto desde las instalaciones del propio Cesga como desde fuera, se puso accesible durante los meses de marzo, abril y mayo a todo el PDI de las universidades gallegas desde la siguiente URL: http://observatorioel.cesga.es/ enquisa/ y se envió un correo electrónico a todos los PDI de las universidades solicitándoles que lo cubriesen. Esta herramienta se diseñó de modo que no pudieran acceder a él los buscadores de Internet evitando de esto modo que pudiera ser cubierto fácilmente por cualquier persona.

Con la herramienta diseñada y en funcionamiento, se envió una notificación para solicitar la cumplimentación del cuestionario online al PDI de las tres universidades. Para ello, en el mes de marzo de 2005, las tres integrantes de la mesa de expertos del proyecto, envió un e-mail utilizando un modelo previamente acordado, a las correspondientes listas de distribución de PDI de cada universidad. A comienzos del mes de abril, se realizó un nuevo envío de emails a todos los docentes, pero esta vez no se utilizaron las listas de distribución, puesto que estas no contenían a todo el profesorado universitario que tenía dirección de correo electrónico, sino que se realizó un envío por e-mail directo a todo el PDI con correo electrónico público accesible desde las páginas Web de las tres universidades. En el mes de mayo se hizo necesario realizar un último recordatorio de cumplimentación del cuestionario en las Universidades de Vigo y A Coruña debido a que no se contaba con la suficiente respuesta que nos asegurara la representativitad de la muestra. A comienzos del mes de junio se dio por finalizada esta fase y se comenzó el tratamiento de los datos obtenidos.

\section{Técnicas e instrumentos para investigar los órganos directivos}

En este caso, al ser un número muy reducido la población a investigar, se optó por una metodología cualitativa. De este modo, se eligió entrevistar a aquellos órganos directivos relacionados directamente o vinculados a las tomas de decisiones estratégicas sobre la introducción y utilización de TIC en la universidad, de tal modo que nos permitiera investigar las políticas y el nivel de implicación de las universidades en el desarrollo del e-learning. Para la investigación de este colectivo se diseñó un protocolo de entrevista semiestructurada. Para la elaboración de este protocolo, así como para la identificación de la muestra, se tuvo en cuenta el análisis de fuentes públicas documentales extraídas de las páginas Web de cada universidad, relativa tanto a aquellos Vicerrectorados implicados en políticas de integración de las TIC en la enseñanza, y más concretamente los que estaban directamente vinculados a los Campus Virtuales de sus Universidades, como a las dimensiones del cuestionario elaborado para el profesorado. El protocolo elaborado recopiló información en torno a las siguientes ideas eje: 
- Perfil.

- Fomento de las TIC en la educación.

- Barreras que impiden la utilización de las TIC en la enseñanza.

- Planificación.

La realización de las entrevistas se llevó a cabo por las integrantes de la mesa y por técnicos del Cesga. La presentación de la entrevista se efectuó por correo electrónico.

\begin{tabular}{|c|c|}
\hline \multicolumn{2}{|c|}{ Instrumentos utilizados } \\
\hline Fuentes públicas documentales. & Páginas Web de las Universidades. \\
\hline Entrevista Semiestructurada & \\
\hline
\end{tabular}

Tabla 6: Instrumentos utilizados para investigar los Órganos Directivos.

Todas las entrevistas fueron realizadas en persona y se grabaron para su posterior transcripción.

Técnicas e instrumentos para investigar los Administradores y Asesores pedagógicos de los Campus Virtuales

En el caso de los administradores de los campus virtuales de las universidades, realizaron entrevistas semiestructuradas. Para la elaboración del protocolo de esta entrevista y para la identificación de la muestra, se tuvo en cuenta tanto el análisis de fuentes documentales realizado de la Web de cada universidad relativa a los campus virtuales como las dimensiones del cuestionario elaborado para el profesorado.

\section{Instrumentos utilizados}

Páginas Web de los Campus Virtuales de las universidades.

Fuentes públicas documentales.

Memorias anuales de actividades de los Campus Virtuales.

Entrevista Semiestructurada.

Tabla 7: Instrumentos utilizados para investigar los Administradores de los Campus Virtuales. 
De esta forma obtuvimos, por una parte, información acerca del perfil, actividad, etc. de los propios administradores y asesores pedagógicos de los Campus Virtuales, así como acerca de la utilización del Campus Virtual por el propio profesorado, dotando así al proceso de investigación de continuidad, coherencia y por consiguiente permitiendo la relación, triangulación y cotejo entre datos procedentes de instrumentos y metodologías cuantitativas y cualitativas. El protocolo elaborado recopiló información en torno a las siguientes ideas eje:

- Recursos humanos: aspectos personales, académicos y profesionales de los administradores y asesores pedagógicos de los Campus Virtuales.

- Recursos Técnicos Software: tipo de recursos software utilizados en los Campus Virtuales.

- Recursos Técnicos Hardware: recursos tecnológicos hardware utilizados en los Campus Virtuales.

- Servicios: servicios que oferta el Campus Virtual a la comunidad educativa.

- Utilización de las TIC: grado de utilización por el profesorado del Campus Virtual, de las materias y de las modalidades de enseñanza que utilizan.

- Formación en TIC: formación que posee el profesorado en la utilización de TIC's en la enseñanza.

- Contenidos: características y tipo de contenidos que utilizan los docentes en su trabajo en los Campus Virtuales.

- Fomento de las TIC: aspectos que fomentan o inhiben la utilización de los Campus Virtuales por parte de los docentes.

- Perspectivas de futuro: opiniones de los administradores de los Campus Virtuales relativas a las líneas futuras de desarrollo de estos entornos.

La realización de las entrevistas se llevó a cabo por las integrantes de la mesa, por una investigadora colaboradora de la Universidad de Vigo y técnicos del Cesga. La presentación de la entrevista se efectuó por correo electrónico.

Todas las entrevistas fueron realizadas en persona y se grabaron para su posterior trascripción.

La realización de las entrevistas se efectuó durante los meses de abril y mayo de 2005, coincidiendo con el período en el que estaba online el cuestionario de investigación para el profesorado. 
Técnicas e instrumentos para estudiar los Grupos de Investigación en e-learning.

De la misma forma que en los casos anteriores, se optó por una metodología de corte cualitativo, mediante el análisis de fuentes públicas documentales y la realización de entrevistas semiestructuradas.

\begin{tabular}{|l|l|}
\hline \multicolumn{2}{|c|}{ Instrumentos utilizados } \\
\hline Fuentes públicas documentales: & $\begin{array}{l}\text { Páginas Web de las OTRI. } \\
\text { Páginas Web de los Grupos de Investigación. }\end{array}$ \\
\hline Entrevista Semiestructurada. & \\
\hline
\end{tabular}

Tabla 8: Instrumentos utilizados para analizar los grupos de investigación en e-learning.

Por lo que se refiere a fuentes públicas documentales consultadas en las universidades y como paso previo a elaboración del protocolo de entrevista, se identificaron a través de los portales Web de las Oficinas de Transferencia de Resultados e Investigación (OTRI) de cada Universidad, aquellos grupos que habían realizado durante los últimos tres años o estaban realizando en la actualidad investigaciones relacionadas con e-learning. Esto nos permitió identificar los grupos de investigación a los que realizar más adelante las entrevistas. En este sentido, como se puede ver en la tabla poblacional y muestral correspondiente a los grupos de investigación, a través de las páginas Web de las OTRI de las tres universidades gallegas se identificaron un total de 19 grupos que realizaban estudios sobre e-learning o tenían/habían tenido alguna relación directa con esta actividad. Para identificarlos se tuvo en cuenta no sólo las líneas principales de investigación de cada grupo, sino también las palabras clave que describen la actividad que realizan.

Posteriormente se elaboró un protocolo de entrevista semiestructurada atendiendo tanto a la información disponible a tal efecto en la página Web de las OTRI de cada Universidad, como a las dimensiones del Módulo de Investigación correspondiente al cuestionario elaborado para el profesorado. De esta forma dotamos al proceso de investigación de continuidad, coherencia y por consiguiente permitiendo la relación, triangulación y cotejo entre datos procedentes de instrumentos y metodologías cuantitativas y cualitativas. El protocolo elaborado recopiló información en torno a las siguientes ideas eje: 
- Perfil del grupo de investigación: aspectos personales, académicos y profesionales de los grupos de investigación en e-learning de las universidades.

- Servicios: servicios que ofertan los grupos que investigan e-learning en la universidad.

- Contenidos: se analiza tanto la oferta como la demanda de contenidos o cursos por parte de los grupos de investigación, así como las previsiones futuras de ofertar formación a través de e-learning.

- Recursos Técnicos: se analiza el tipo de recursos técnicos que oferta y demanda cada grupo de investigación, así como aquellos de los que necesita para investigar pero no dispone de ellos.

- Investigación: medidas que fomentan la investigación en e-learning, las barreras que limitan su desarrollo, las líneas y proyectos de investigación llevados a cabo en los tres últimos años, las líneas futuras de investigación, la inversión económica que hacen en e-learning estos grupos, el estado en el que se encuentra actualmente la investigación en e-learning en Galicia y las conclusiones más importantes obtenidas sobre e-learning de los proyectos de investigación realizados.

Las entrevistas las realizaron las integrantes de la mesa de expertos, una investigadora colaboradora de la Universidad de Vigo y técnicos del Cesga. La presentación de la entrevista se efectuó por correo electrónico.

La mayoría de las entrevistas fueron realizadas en persona y se grabaron para su posterior trascripción. El resto fueron remitidas por correo electrónico, para ajustarse a los plazos del proyecto.

\section{Temporalización de la investigación}

A continuación se puede ver detallada, acorde a los ámbitos objeto de estudio, esto es, universidad y empresa, la temporalización del proceso investigador que se ha llevado a cabo durante el desarrollo de este proyecto.

\section{Temporalización de la investigación en la universidad}

Teniendo en cuenta el calendario del proyecto (septiembre de 2004 - diciembre de 2005), las acciones llevadas a cabo en la universidad se estructuraron de la siguiente manera: 
- Análisis de fuentes públicas documentales correspondiente a la información disponible sobre e-learning en las páginas web de las Universidades (Noviembre 2004-Febrero 2005).

- Elaboración del cuestionario para Personal docente e investigador (EneroFebrero 2005).

- Elaboración del cuestionario online para PDI (Febrero-Marzo 2005).

- Puesta online del cuestionario para el personal docente e investigador (Marzo-Mayo 2005).

- Elaboración de protocolos de entrevistas para Órganos directivos, administradores de los Campus Virtuales, asesores pedagógicos de los Campus Virtuales y grupos de investigación en e-learning (Marzo 2005).

- Realización de entrevistas a órganos directivos, administradores de Campus Virtuales y grupos de investigación (Abril y Mayo 2005).

- Análisis de datos del cuestionario de PDI (Junio-Agosto 2005).

- Informe de buenas prácticas de e-learning en la universidad (SeptiembreOctubre 2005).

- Análisis de datos de las entrevistas realizadas a Órganos directivos, administradores de los Campus Virtuales, asesores pedagógicos de los Campus Virtuales y grupos de investigación en e-learning (Octubre 2005).

- Elaboración del informe final de investigación (Junio-Diciembre 2005).

- Elaboración de conclusiones (Diciembre 2005).

En el caso de la universidad, el ámbito temporal de la investigación se centra en los últimos tres cursos académicos, esto es, 2002-2005.

\section{NOTAS}

1. Tamaño de la población.

2. Porcentaje de la población.

3. Tamaño de la muestra.

4. Porcentaje de la muestra.

5. Población total de Personal Docente e Investigador de las tres universidades gallegas en el curso académico 2004-2005. 


\section{REFERENCIAS BIBLIOGRÁFICAS}

Anido Rifón, L. (2003). Informe para a implantación dun Sistema de Teleformación na Universidade de Vigo. Área de Innovación Académica. Vicerrectorado de Posgrado e Formación Permanente. Universidad de Vigo. [en línea]. Disponible en: http://apv.uvigo. es/apv/docs/docs_memoria/informe. pdf [consulta 2004, 7 de marzo]

Área de Posgrado Virtual (2004). Memoria del curso 2003/2004. Vicerrectorado de Titulacións, Posgrao e Formación Permanente. Universidad de Vigo. [en línea]. Disponible en: http://apv. uvigo.es/apv/docs/docs_memoria/ memoria_2003_2004.pdf $\quad$ [consulta 2005, 17 de mayo]

Barro Ameneiro, S. (2004). Las Tecnologías de la Información y las Comunicaciones en el Sistema Universitario Español. Santiago de Compostela: Conferencia de Rectores de las Universidades Españolas.

Bisquerra, R. (1989). Métodos de investigación educativa. Guía práctica. Barcelona: CEAC.

Cabero, J. (Ed.). (2000). Nuevas tecnologías aplicadas a la educación. Madrid: Síntesis.

Cabero Almenara, J. (dir). (2002). Las TIC's en la Universidad. Sevilla: Editorial MAD.

Cabero Almenara, J. (dir.) (2003): Las nuevas tecnologías en la actividad universitaria. PixelBit, 20, 81-100.

Cebreiro López, B. y Fernández Morante, C. (2004). Novas Tecnoloxías na educación, en: Santos Rego, M. A. (dir.) A investigación Educativa en Galicia (1989-2001). (Tomo 2), Santiago de Compostela: Consellería de Educación e Ordenación Universitaria, 124-169.

Cebreiro López, B.; Fernández Morante, C. (2003). Las Tecnologías de la Comunicación en el espacio europeo para la educación superior, Comunicar. Revista Científica Iberoamericana de Comunicación y Educación, 21, 57-61.

Centro de Tecnologías para a Aprendizaxe (CeTA) (2004). Memoria de actividades
2003-2004. Vicerreitorado de Terceiro Ciclo e Extensión Docente. Universidad de Santiago de Compostela. [en línea]. Disponible en: http://www.usc.es/ceta/ normativa/Memoria_CeTA2003-2004. pdf [consulta2005, 17 de octubre]

Duart, J. M.; Lupiáñez, F. (2005). E-strategias en la Introducción y uso de las TIC en la Universidad, en: Duart, J. M.; Lupiáñez, F. (coords.). Las TIC en la universidad: estrategias y transformación institucional [monográfico en línea]. Revista de Universidad y Sociedad del Conocimiento (RUSC). 2 (1) [en línea]. Disponible en: http://www.uoc.edu/ rusc/dt/esp/duart0405.pdf [consulta 2005, 17 de octubre]

Fernández Morante, C. (2002a). Los medios audiovisuales, informáticos y nuevas tecnologías en los centros educativos gallegos: presencia y usos. Santiago de Compostela: Servicio de Publicaciones de la Universidad de Santiago.

Fernández Morante, C.; Cebreiro, B. (2002b). La preparación de los profesores para el dominio técnico, didáctico y el diseño/ producción de medios en Galicia. Innovación Educativa, 12, 109-122.

Fernández Morante, C.; Cebreiro, B. (2002c). La integración de los medios y nuevas tecnologías en los centros y prácticas docentes. Pixel-Bit, 20, 33 - 42.

Fernández Morante, C.; Cebreiro, B. (2001a). Los medios audiovisuales, informáticos y NTIC en Galicia: ¿de qué disponen los profesores, Adaxe, 17, 247-267.

Fernández Morante, C.; Cebreiro, B. (2001b). Los medios en los centros educativos gallegos: elementos organizativos que ayudan o dificultan su integración. Adaxe, 17, 227-246.

García Tobío, J.; Soto Carballo, J.; Gomes de Sousa, A. P.; Touriñán López, J. M. (2005). El concepto de Educación a Distancia: e-learning, m-learning, b-learning. El potencial de Internet y las Redes informáticas, en: Touriñán López, J. M. (Dir.) (2005). Educación electrónica. El reto de la sociedad digital en la 
escuela. Santiago de Compostela. Xunta de Galicia. Consellería de Educación e Ordenación Universitaria.

Gromaz Campos, M.; Arribi Vilela, J.; Fernández Morante, C. (2004). E-Learning: metodologías, tecnologías y tendencias. II Congreso online del Observatorio para la Cibersociedad. [en línea]. Disponible en: http://www.cibersociedad.net/ congres2004/grups/fitxacom_publica2. php?grup=18\&id = 229\&idioma $=e s$ [consulta 2005, 17 de enero]

Gromaz Campos, M. y otros (2005). El Observatorio gallego de e-learning, en: I Congreso Ibérico sobre la Fractura Digital. Las Tecnologías de la Información y de la Comunicación: Retos para la Educación del Futuro. Silleda.

Marcelo, C. (Coord) (2002). E-LearningTeleformación. Barcelona. Ediciones Gestión 2000.

Marchesi, Á. y otros. Tecnología y aprendizaje. Investigación sobre el impacto del ordenador en el aula. [en línea]. Disponible en: http://www. librosvivos.org/piloto/ [consulta 2005, 17 de octubre]

Observatorio gallego de e-learning. [en línea]. Disponible en: http://observatorioel. cesga.es/ [consulta 2005, 10 de octubre].

Observatorio gallego de e-learning. Directorio de grupos de investigación en e-learning . [en línea]. Disponible en: http:// observatorioel.cesga.es/universidades [consulta 2005, 10 de octubre]

Rodríguez Malmierca, M. J.; Gromaz Campos, M. (2001). La Tele-Enseñanza en el Centro de Supercomputación de Galicia. Quaderns Digitals, 22. [en línea]. Disponible en: http://216.12.215.150/ index.php?accionMenu=hemeroteca. VisualizaArticuloIU.visualiza\&articulo_ id=192 [consulta 2004, 17 de octubre]

Rodríguez Malmierca, M. J.; Gromaz Campos, M. (2002). Modelo Psicopedagógico de las Aulas de Tele-Enseñanza del Centro de Supercomputación de Galicia. PíxelBit, 18. [en línea]. Disponible en: http:// www.sav.us.es/pixelbit/articulos/n18/ n18art/art189.htm [consulta 2004, 7 de marzo]

Rodríguez Malmierca, M. J.; Gromaz Campos, M. (2004). E-Learning colaborativo en un Sistema de Gestión del Aprendizaje: Los Grupos de Trabajo Virtuales. Fórum Aula y TIC. Alicante. Instituto de Ciencias de la Educación. Universidad de Alicante.

Rodríguez Malmierca, M. J; Gromaz Campos, M.; Rubio Prieto, M. A.; Rubio Gayo, B. (2004). Tecnología y Posibilidades de los Nodos Access Grid. [en línea]. Disponible en: http://torga.net.ccg.pt/parceiros/ contents / Documentos/Tarefas/ TorgaNet-T1.3-D1.3-NCL_CESGA-2004o.o.pdf [consulta 2005, 13 de octubre]

Salinas, J. (2004). Innovación docente y uso de las TIC en la enseñanza universitaria. Revista de Universidad y Sociedad del Conocimiento (RUSC). UOC. 1 (1) [en línea]. Disponible en: http://www. uoc.edu/rusc/dt/esp/salinas1104.pdf [consulta 2005, 13 de octubre]

Sancho i Vinuesa, T. (2004). Internet y la Red de Universidades Catalanas. Barcelona: Universitat Oberta de Catalunya. [en línea]. Disponible en: http://www.uoc. edu/in3/pic/esp/pic6.html/ [consulta 2005, 07 de febrero]

Unidad de Teleformación de la Universidad de A Coruña. [en línea]. Disponible en: http://www.udc.es/uep/ [consulta 2004, 27 de diciembre]

Universidad de Santiago de Compostela. Plan de Calidad. [en línea]. Disponible en: http://www.usc.es/ calidade/ PlanCalidade_galego.pdf [consulta 2005, 10 de octubre]

Universidad de Santiago de Compostela. Plan estratégico de las Tecnologías de la Información y las Comunicaciones 20042006. [en línea]. Disponible en: http:// www.usc.es/atic/PETIC.pdf [consulta 2005, 10 de octubre]

Universidad de Vigo. Plan de Calidad Docente. [en línea]. Disponible en: http://webs.uvigo.es/webcalidad/area calidad/documentos/plan_cal_doc/ PLAN DE CALIDADE DOCENTE-C. Xob.Dic.2004.pdf [consulta 2005, $10 \mathrm{de}$ octubre] 


\section{PERFIL ACADÉMICO Y PROFESIONAL DE LOS AUTORES}

Manuel Gromaz Campos. Especialista universitario en modelos y sistemas de Tele-Enseñanza. Forma parte del Consejo de Redacción de las revistas DIM y Textos para la Cibersociedad. Profesor del Master e-Learning: TIC para la educación y la formación de la Universidad de Santiago de Compostela y Miembro del consejo científico de ARENOTECH. Actualmente Técnico de e-learning en el Centro de Supercomputación de Galicia (CESGA).

María José Rodríguez Malmierca. Coordinadora Área e-learning. Centro de Supercomputación de Galicia.

Carmen Fernández Morante. Profesora del Dpto. de Didáctica y Organización Escolar. Universidad de Santiago de Compostela.

Beatriz Cebreiro López. Subdirectora CETA. Universidad de Santiago de Compostela.

Miguel Beremejo Paradela. Técnico del Centro de Supercomputación de Galicia.

María Isabel Doval Ruíz. Profesora de Nuevas Tecnologías. Universidad de Vigo.

Marta Fernández Prieto. Profesora de Nuevas Tecnologías. Universidad de A Coruña.

Javier García Tobío. Director Gerente del Centro de Supercomputación de Galicia.

DIRECCIÓN DE LOS AUTORES

Centro de Supercomputación de Galicia (CESGA).

Avda. de Vigo S/N - 15705

Santiago de Compostela

http://www.cesga.es

Fecha de recepción del artículo: 14/06/06

Fecha de aceptación del artículo: 12/12/06 